\title{
Actitudes de los estudiantes universitarios hacia el aprendizaje de la física
}

\author{
Attitudes of university students towards the learning of physics
}

\author{
Julio César Guevara Injoque ${ }^{1}$
}

\section{Resumen}

El propósito de esta investigación es indagar las actitudes hacia el aprendizaje de la física de los estudiantes de diferentes especialidades en el curso de física. La muestra no probabilística estuvo conformada por estudiantes de ambos sexos a quienes se les aplicó la Escala de Actitudes hacia el Aprendizaje de la Física. Los resultados mostraron que hay una actitud favorable de los estudiantes hacia el aprendizaje de la física. La prueba de U de Mann Whitney mostró que los hombres y mujeres tienen actitudes similares hacia el aprendizaje de la física. Con la prueba de KruskalWallis se encontró la existencia de una relación entre las actitudes hacia el aprendizaje de la física y sus especialidades. Se concluye que las actitudes de los estudiantes son positivos hacia el aprendizaje de la física y que esta actitud varía en función de la especialidad del estudiante.

Palabras claves: actitud; actitudes hacia el aprendizaje de la física; física; género.

\begin{abstract}
The purpose of this research is to investigate the attitudes towards physics learning of students of different careers in the course of physics. The nonrandom sample consisted in students of both sexes who were administered the Attitudes towards Physics Learning Scale. The results showed that there is a student favorable attitude towards physics learning. The U de Mann Whitney showed that men and women similar attitudes towards physics learning. With Kruskal- Wallis test, it found the existence of a relationship between attitudes towards physics learning and careers. Thus, the attitudes of students are positive towards physics learning and that this attitude varies depending on the careers of the student.
\end{abstract}

Keywords: attitude; attitudes towards physics learning; physics; gender.

\section{Introducción}

La actitud es un componente en todas las materias de aprendizaje y tienen una posición importante en el proceso de aprendizaje, guiando lo cognitivo, afectivo y conductual del aprendizaje de cualquier unidad pedagógica, en este caso la Física. De acuerdo a Rosenberg y Hovland (1960) las actitudes son predisposiciones a responder a alguna clase de estímulo con ciertas clases de respuesta y están conformadas por tres componentes: el afectivo, el cognitivo y el componente conductual. Conforme con Hendrickson (1997), las actitudes son una forma de estimar el éxito de los estudiantes. Los procesos de enseñanza-aprendizaje deben ser planificadas, organizadas e implementadas para que los estudiantes pueden desarrollar una actitud más positiva (Pintrich \& Schunk (1996).

Eryilmaz et al. (2011) concluyen que una alta motivación hacia la clase de física, se corresponde a una mayor actitud hacia la física y una baja motivación indica una menor actitud hacia la física. Para Adedayo (2011) los factores relacionados que influyen en la actitud de los estudiantes hacia la física es la impresión de que esta es difícil, la falta de interés en aprenderla por considerarla muy tediosa, y por la cantidad de estudiantes en el aula durante la clase de física. Por otro lado, un bajo conocimiento en matemáticas influye en las actitudes y rendimiento hacia la física. Vahedi y Yari (2014) indican que existe una correlación positiva y significativa entre las actitudes hacia física y el conocimiento previo de la matemática. Guzel (2004) muestra que hay una relación entre las actitudes hacia las matemáticas y el rendimiento en física.

En carreras como las ingenierías, un curso de física es importante para su desarrollo y aplicación. Por ende, en el diseño curricular de las ingenierías la física representa la base de otras asignaturas, de tal manera que el alumnado debe cursarla previamente. En consecuencia, la formación de actitudes ha adquirido una particular vigencia en el campo de la educación.

En el ámbito nacional existe poca investigación relacionada a las actitudes hacia la física. Es ahí, la importancia de explorar las actitudes hacia la física del estudiantado de ingeniería, con el propósito de entender y analizar los componentes que subyacen en ellas. La información adquirida puede ser muy importante para introducir cambios encaminados a mejorar el proceso de enseñanza aprendizaje de esta materia. Por lo tanto, esta investigación tiene por objetivo describir las actitudes hacia el aprendizaje de la física en estudiantes universitarios, y su relación con el sexo y la especialidad de los estudiantes matriculados en el curso de Física General. 


\section{Métodos y materiales}

\section{Participantes}

Los participantes fueron 363 estudiantes regulares de las diferentes especialidades que estaban matriculados en el curso de Física General. Esta muestra de conveniencia incluyó a 142 (55,91\%) mujeres y 112 (44,61\%) hombres, con una edad comprendida entre 18 y 26 años $(M=19,36$; $D E=1,71)$. Se excluyeron los estudiantes que faltaron el día de la aplicación de la encuesta.

\section{Instrumento}

La actitud hacia el aprendizaje de la física se midió con la escala de Actitudes hacia el aprendizaje de la física (Anexo 1), la cual mide la actitud que tienen los estudiantes hacia el aprendizaje de la física. La escala fue desarrollada para la presente investigación tomando en cuenta el modelo tridimensional de la actitud propuesto por Rosenberg y Hovland (1960), los cuales postulan que la actitud está conformada por tres componentes: cognitivo, afectivo y conductual. El instrumento estuvo conformado por 22 enunciados acerca de la actitud hacia el aprendizaje de la física que se calificaban de acuerdo a una escala tipo Likert con cinco opciones $(1=$ totalmente en desacuerdo, 5 = totalmente de acuerdo) y con tres dimensiones - cognitivo, afectivo y conductual -. El componente cognitivo está conformado por diez ítems y hace referencia a lo que el estudiante piensa y cree acerca de aprendizaje hacia la física. El componente afectivo está conformado por ocho ítems y alude a los sentimientos positivos y negativos del estudiante hacia el aprendizaje hacia la física. El componente conductual tiene cuatro ítems e indica las acciones favorables y desfavorables del estudiante hacia el aprendizaje hacia la física. Las propiedades psicométricas de esta escala, Tabla 1, indica que la dimensiones cognitiva, afectiva y conductual presentan correlaciones ítems test corregida (citc) superiores a 0,20 (Kline, 2011) lo cual aporta evidencias de validez de contenido (Santisteban, 2009).

Tabla 1. Validez de contenido y fiabilidad de la escala de actitudes hacia el aprendizaje de la física

\begin{tabular}{lcccc}
\hline & $\begin{array}{c}\text { Ítems } \\
\text { Cognitivo }\end{array}$ & $\begin{array}{c}\text { citc } \\
\text { mínimo }\end{array}$ & $\begin{array}{c}\text { citc } \\
\text { máximo }\end{array}$ & $\alpha$ \\
\cline { 2 - 5 } Afectivo & 10 & 0,56 & 0,78 & 0,91 \\
Conductual & 8 & 0,56 & 0,75 & 0,89 \\
Escala total & 22 & 0,34 & 0,63 & 0,76 \\
\hline
\end{tabular}

$\alpha$ : Alfa de Cronbach; citc: correlación ítems test corregida.

Para la validez de constructo del instrumento se utilizó el análisis factorial confirmatorio mediante el modelo de ecuaciones estructurales (Byrne, 2013). Siguiendo a Hu y Bentler (1999) para un buen ajuste del modelo se tomó en consideración los siguientes índices $\chi^{2} / \mathrm{gl}<3$, RMSE es menor a 0,08 y los índices TLI y CFI deben ser mayores a 0,90, Tabla 2. Como resultado de este análisis se confirmó que la escala de actitudes hacia el aprendizaje de la física está conformada por los tres factores propuestos. Además la fiabilidad de la escala fue de 0,92 medido a través del alfa de Cronbach lo cual es alta según considera Hair et al. (2010).

Tabla 2. Índices de ajustes obtenidos para la escala de actitudes hacia el aprendizaje de la física

\begin{tabular}{lcc}
\hline Índice de ajuste & Valor obtenido & Valor recomendado* \\
\hline$\chi^{2} / \mathrm{gl}$ & 2,23 & $<3$ \\
TLI &, 99 & $\geq, 90$ \\
CFI &, 99 & $\geq, 90$ \\
RMSEA &, 062 & $<, 08$ \\
\hline
\end{tabular}

*Nota: Hu y Bentler, 1999

La actitud hacia el aprendizaje de la física de los estudiantes obtenida por la sumatoria de sus respuestas a los enunciados del instrumento. Los puntajes máximos y mínimos que se puede obtener en esta escala son 110 puntos y 22 puntos respectivamente. Así, puntuaciones altas en la escala se interpretaran como actitudes favorables hacia el aprendizaje de la física.

\section{Diseño}

El enfoque utilizado en esta investigación fue el cuantitativo con un diseño no experimental transversal del tipo descriptivo relacional (Hernández et al., 2010).

\section{Procedimiento}

Los datos fueron recolectados en cada grupo del curso de Física General durante el segundo semestre del 2014 en el primer día de clase y en coordinación con los profesores del curso. Se informó a los estudiantes que estaban presentes en el aula que la investigación tenía una naturaleza académica y era anónima, siendo voluntario participar en el estudio. Luego, se procedió a distribuir la encuesta entre los estudiantes donde primero informaron sobre su edad, sexo y especialidad. Posteriormente respondieron el grado de acuerdo o desacuerdo hacia las preguntas de la escala de actitudes. Al completar la encuesta los estudiantes procedieron a devolverla al profesor y el tiempo que se tomaron en responder, los estudiantes al instrumento, fue de aproximadamente de 15 minutos.

\section{Análisis de datos}

Los datos obtenidos fueron analizados usando el paquete estadístico $\mathrm{R}$ versión 3.1.0. La descripción de los datos se realizó utilizando la estadística descriptiva a través de las medias y desviación estándar. Se usó la prueba de Kolmogorov-Smirnov para constatar la hipótesis de normalidad de los puntajes obtenidos en la escala de actitudes hacia el aprendizaje de la física Tabla 3. A fin de determinar las diferencias entre las puntuaciones de los estudiantes de la muestra obtenidos en la escala de actitudes hacia el aprendizaje de la física y el sexo se aplicó 
la prueba de U de Mann Whitney. Se empleó la prueba de Kruskal-Wallis para determinar las diferencias entre las puntuaciones en la escala de actitudes hacia el aprendizaje de la física de los estudiantes y su especialidad. Además, se aplicó la prueba de Dunn para identificar entre que especialidades existen las diferencias más significativas. El nivel de significancia para las pruebas fue de 0,05 .

\section{Resultados y discusión}

La actitud hacia el aprendizaje de la física fue tratada como variable del tipo cuantitativa, pues cada uno de los estudiantes de la muestra tuvo puntuaciones directas en esta escala. Para interpretar la escala se consideró que los puntajes superiores al puntaje promedio obtenido por los estudiantes de la muestra en la escala son considerados actitudes favorables. La puntuación media obtenida en el cuestionario por los estudiantes de la muestra en el curso de Física General, fue $M=78,19, D E=13,92$, siendo el puntaje menor de 30 y el mayor de 105, Tabla 3. De esta manera aquellos estudiantes que tuvieron una puntuación mayor a 78,19 se han considerado que tenían una actitud favorable hacia el aprendizaje de la física.

Asimismo para las dimensiones cognitiva, afectiva y conductual si las medias eran mayores a 36,5, 28,60 y 12,83 las actitudes fueron consideradas como favorables.

La prueba de Kolmogorov-Smirnov para la normalidad (Field, 2005) indican que la distribución de las puntuaciones de la escala de actitudes hacia el aprendizaje de la física se desvió significativamente de una distribución normal $(\mathrm{D}=0,076 \mathrm{p}<0,05)$. Por tanto, se determinó utilizar pruebas no paramétricas.

Tabla 3. Estadísticos descriptivos para la escala de actitudes hacia el aprendizaje de la física y sus dimensiones.

\begin{tabular}{|c|c|c|c|c|c|c|}
\hline & $n$ & Rango & Mínimo & Máximo & $M$ & $D E$ \\
\hline Cognitivo & 363 & 36 & 13 & 49 & 36,75 & 6,98 \\
\hline Afectivo & 363 & 32 & 8 & 40 & 28,60 & 6,61 \\
\hline Conductual & 363 & 15 & 4 & 19 & 12,83 & 2,03 \\
\hline $\begin{array}{l}\text { Actitudes } \\
\text { hacia el } \\
\text { aprendizaje } \\
\text { de la física }\end{array}$ & 363 & 75 & 30 & 105 & 78,19 & 13,92 \\
\hline
\end{tabular}

La Tabla 4, muestra el porcentaje de estudiantes que tuvieron una actitud favorable y desfavorable hacia el aprendizaje de la física. En general tanto para la escala total como para cada dimensión, los estudiantes participantes mostraron actitudes favorables $(55,1 \%)$ hacia el aprendizaje de la física. Así mismo, en la Tabla 5 se observa que en las tres dimensiones los estudiantes mostraron actitudes favorables siendo las dimensiones conductual $(59,0 \%)$ y cognitiva $(58,1 \%)$ donde se alcanzó los mayores porcentajes.
Tabla 4. Actitudes hacia el aprendizaje de la física de los estudiantes

\begin{tabular}{lcccc}
\hline & \multicolumn{2}{c}{ Favorable } & \multicolumn{2}{c}{ Desfavorable } \\
\cline { 2 - 5 } & $n$ & $\%$ & $n$ & $\%$ \\
\hline Cognitivo & 211 & 58,1 & 152 & 41,9 \\
Afectivo & 196 & 54,0 & 167 & 46,0 \\
Conductual & 214 & 59,0 & 149 & 41,0 \\
Actitudes hacia el aprendizaje de la & 200 & 55,1 & 163 & 44,9 \\
física & & & & \\
\hline
\end{tabular}

Por otro lado, la Tabla 5 muestra que las estudiantes mujeres mostraron una mejor actitud $(M=78,22 ; D E=$ $0,84)$ hacia el aprendizaje de la física que lo estudiantes hombres $(M=78,15 ; D E=1,29)$.

Tabla 5. Actitudes hacia el aprendizaje de la física de los estudiantes por sexo

\begin{tabular}{lccccccccc}
\hline & \multicolumn{3}{c}{$\begin{array}{c}\text { Actitudes } \\
\text { hacia el } \\
\text { aprendizaje } \\
\text { de la física }\end{array}$} & Cognitivo & Afectivo & Conductual \\
\cline { 2 - 9 } & $n$ & $M$ & $D e$ & $M$ & $D e$ & $M$ & $D e$ & $M$ & $D e$ \\
\hline Mujer & 208 & 78,22 & 0,84 & 37,19 & 0,41 & 28,5 & 0,14 & 12,79 & 0,14 \\
Hombre 155 & 78,15 & 1,29 & 36,17 & 0,66 & 29,09 & 0,59 & 12,89 & 0,16 \\
\hline N=363 & & & & & & & &
\end{tabular}

Luego, se examinó la relación entre las actitudes hacia el aprendizaje de la física y el sexo de los estudiantes. En la Tabla 6, se observa el resultado de la prueba U de Mann Whitney, mostrando que las actitudes hacia el aprendizaje de la física de los estudiantes hombres ( $M d$ $=82) \mathrm{y}$ de las estudiantes mujeres $(M d=79)$ no difieren significativamente $z=-1,09 ; p=0,28 ; r=0,06$. Sin embargo, se encontró una diferencia significativa en la dimensión afectiva entre hombres $(M d=31)$ y mujeres $(M d=28), z=-2,22 ; p=0,03 ; r=0,12$.

Tabla 6. Relación entre las Actitudes hacia el aprendizaje de la física de los estudiantes y sexo

\begin{tabular}{|c|c|c|c|c|c|}
\hline & & Mujer & & ombre & \\
\hline & $M d$ & $\begin{array}{l}\text { Rango } \\
\text { medio }\end{array}$ & $M d$ & $\begin{array}{l}\text { Rango } \\
\text { medio }\end{array}$ & z \\
\hline Cognitivo & 35,5 & 182,70 & 38 & 181,06 & $-0,15$ \\
\hline Afectivo & 28 & 171,44 & 31 & 196,17 & $-2,22^{*}$ \\
\hline Conductual & 13 & 179,88 & 13 & 184,85 & $-0,45$ \\
\hline $\begin{array}{l}\text { Actitudes hacia el aprendizaje } \\
\text { de la física }\end{array}$ & 79 & 176,81 & 82 & 188,96 & $-1,09$ \\
\hline
\end{tabular}
$* p<0,05$

En la Tabla 7, se observa la puntuación media obtenida por los estudiantes en la escala de actitudes hacia el aprendizaje de la física por especialidad. Se encontró que los estudiantes de Agrícola tuvieron una actitud más favorable $(M=83,51 ; D E=2,10)$ hacia el aprendizaje de la física y los estudiantes de Estadística fueron los que 
tuvieron la actitud más desfavorable $(M=69,79 ; D E=$ $3,07)$ hacia el aprendizaje de la física.

Por otro lado, se utilizó la prueba de Kruskal-Wallis para identificar si había diferencias en las actitudes hacia el aprendizaje de la física con las especialidades de los estudiantes. Así pues, se encontró diferencias entre las actitudes de los estudiantes y sus especialidades $(\mathrm{H}$ $(11)=32,69 ; p=0,001)$. Para identificar entre cuales especialidades existe diferencias se aplicó la prueba de Dunn con una corrección de Bonferroni. El resultado de esta prueba indicó que hubo una diferencia significativa entre las actitudes de los estudiantes de Agrícola $(M d=$ $87)$ y los estudiantes de Estadística $(M d=69,5)$ hacia el aprendizaje de la física.

Tabla 7. Actitudes hacia el aprendizaje de la física de los estudiantes del curso de Física General por especialidad

\begin{tabular}{lccc}
\hline Especialidad & $n$ & $M$ & $D E$ \\
\hline Agronomía & 78 & 76,00 & 1,43 \\
Biología & 23 & 80,96 & 2,58 \\
Ambiental & 16 & 80,38 & 2,91 \\
Meteorología & 17 & 78,82 & 4,66 \\
Forestal & 23 & 72,91 & 3,58 \\
Economía & 26 & 75,35 & 2,95 \\
Estadística & 14 & 69,79 & 3,07 \\
Gestión & 24 & 73,04 & 2,34 \\
Agrícola & 35 & 83,51 & 2,10 \\
Zootecnia & 30 & 79,51 & 2,59 \\
Pesquería & 39 & 82,05 & 1,88 \\
Alimentaria & 38 & 81,39 & 2,52 \\
\hline
\end{tabular}

El propósito de esta investigación fue describir las actitudes de los estudiantes hacia el aprendizaje de la física, y como estas actitudes se relacionaban con su especialidad y sexo. Con relación a las actitudes hacia el aprendizaje de la física, los estudiantes mostraron una actitud favorable por aprender física. Este resultado indica que los estudiantes creen que aprender física es útil e interesante además de ser necesario. Así mismo, hay un agrado por aprender física por considerarla importante para su formación. Este resultado concuerda con el trabajo de Olasimbo (2012) quien encontró que las actitudes de los estudiantes eran favorables hacia la física.

Por otro lado, se encontró que no existe una relación entre el sexo de los estudiantes y sus actitudes hacia el aprendizaje de la física. Es decir, tanto hombres como mujeres tienen actitudes similares hacia la física coincidiendo con Shaw (2003) y Pell \& Jarvis (2005) y Kaya y Böyük (2011). Este resultado no coincide con los resultados de Veloo et al. (2015) y Fatoba y Aladejana (2014) quienes hallaron diferencias en las actitudes hacia la física entre hombres y mujeres.

Con respecto a la especialidad del estudiante y su actitud hacia el aprendizaje de la física, se encontró que son los estudiantes de Agrícola los que muestran actitudes más favorables el aprendizaje de la física y que los estudiantes de Estadística expresan la actitud más baja hacia el aprendizaje de la física. Además, no se encontró diferencias significativas entre las actitudes de las demás especialidades. Este resultado debe tomarse en cuenta al momento de desarrollar las sesiones de enseñanza aprendizaje.

\section{Conclusión}

Las actitudes hacia el aprendizaje de la física son importantes para su estudio, ya que es considerada como una ciencia difícil de aprender por la mayoría de estudiantes. Los resultados de este estudio sugieren que los estudiantes tienen en su mayoría actitudes favorables hacia el aprendizaje de la física; lo cual debe ser tomado en cuenta al momento de desarrollar los procesos de enseñanza-aprendizaje. Así pues, tanto los estudiantes hombres como mujeres han mostrado actitudes similares hacia el aprendizaje de la física. Este hecho facilita la presentación del material de aprendizaje independientemente del sexo de los estudiantes. Sin embargo se observó que los hombres tienen un mayor afecto hacia el aprendizaje de la física. Por último, se halló que los estudiantes de Agrícola tienen una mejor actitud para aprender física que los estudiantes de Estadística. Esto deberá considerarse para potenciar los procesos de aprendizajes en estos estudiantes.

Estos resultados expresan la importancia de tomar en cuenta las actitudes al momento de enseñar. Así, estudiantes con actitudes favorables hacia el aprendizaje de la física tendrán mayores posibilidades de potenciar sus aprendizajes. Por último, futuras investigaciones deberán incluir otras variables como el ambiente de aprendizaje, los estilos de enseñanza de los docentes, los conceptos previos hacia el estudio de la física, rendimiento académico, etc. con relación a las actitudes de los estudiantes hacia el aprendizaje de la física.

\section{Literatura citada}

Adedayo, J. 2011. Analysis of factors influencing students' attitudes towards practical aspect of secondary school physics in Ekiti state. International Journal of Multidisciplinary Research and Development 2(7): 417-421.

Byrne, B. 2013. Structural Equation Modeling with Mplus: Basic Concepts, Applications, and Programming. NJ: Routledge.

Eryilmaz, A.; Yildiz, I. and Akin, S. 2011. Investigating of Relationships between Attitudes towards Physics Laboratories, Motivation and Amotivation for the Class Engagement. Eurasian J. Phys. Chem. Educ. 59-64.

Fatoba, J. \& Aladejana, L. 2014. Effects of gender on students' attitude to physics in secondary schools in Oyo State, Nigeria. European Scientific Journal 10 (7).

Field, A. 2005. Discovering Statistics Using SPSS, 2nd edition. London: Sage.

Guzel, H. 2004. The Relationship between students' 
success in physics lessons and their attitudes towards mathematics. Journal of Turkish Science Education 1(1).

Hair; Black; Babin, \& Anderson. 2010. Multivariate Data Analysis. Seventh Edition. Prentice Hall, Upper Saddle River, New Jersey.

Kaya, H. and Böyük, U. 2011. Attitude towards physics lessons and physical experiments of the high school students, European of Physics Education 2(1).

$\mathrm{Hu}$ and Bentler. 1999. Cutoff criteria for fit indexes in covariance structure analysis: Conventional criteria versus alternatives, Structural Equation Modeling 6(1): 1-55.

Hendrickson, A. 1997. Predicting student success with the learning and study strategies inventory (LASSI). Unpublished Master's Thesis. Iowa State University. $76 \mathrm{p}$.

Hernández, R.; Fernández, C. y Baptista, P. 2010. Metodología de la investigación. México: McGrawHill/Interamericana Editores.

Kline, R. 2010. Principles and practice of structural equation modelling. Third edition. New York: The Guilford Press.

Olasimbo, O. 2012. Attitudes of Students towards the Study of Physics in College of Education Ikere Ekiti, Ekiti State, Nigeria. American International Journal of Contemporary Research 2(12): 86-96.

Pell, T., \& Jarvis, T. 2005. Developing attitude to science scales for use with children of ages from five to eleven years. International Journal in Science Education 23(1): 847 - 862.

Pintrich, P. R. \& Schunk, D. H. 1996. Motivation in education: Theory, Research, and 15. Application. Englewood Cliffs, NJ: Prentice-Hall Inc.

Rosenberg, M. and Hovland, C. 1960. "Cognitive, Affective and Behavioral Components of Attitudes". In Attitude Organization and Change: An Analysis of Consistency among Attitude Components. New Haven: Yale University Press.

Santisteban, C. 2009. Principios de psicometría. Madrid: Síntesis.

Shaw, K. A. 2003. The Development of a Physics SelfEfficacy Instrument for Use in the Introductory Classroom Physics, Astronomy, and Chemistry. Education Research Group Southern Illinois University Edwardsville.

Vahedi, S. and Yari, M. 2014. Role of Cognitive and Emotional Factors on Educational Achievement among High School Students in Physics. European Online Journal of Natural and Social Sciences 3(3): 572-579.

Veloo, A.; Nor, R. and .Khalid, R. 2015. Attitude towards Physics and Additional Mathematics Achievement towards Physics Achievement. International Education Studies 8(3). 


\section{ANEXO}

INSTRUMENTO PARA MEDIR LAS ACTITUDES HACIA EL APRENDIZAJE DE LA FÍSICA

\section{INSTRUCCIONES}

A continuación encontrará una serie de proposiciones. Marca la opción correspondiente al grado de acuerdo o desacuerdo con lo ahí expresado. Recuerda no existe respuesta correcta o incorrecta. Las opciones de respuestas son:

Muchas Gracias.

TOTALMENTE EN DESACUERDO

EN DESACUERDO 2

INDECISO 3

DE ACUERDO 4

TOTALMENTE DE ACUERDO. 5

Sexo:

Edad:

Especialidad:

\begin{tabular}{|c|c|c|}
\hline Dimensión & Ítems & 123 \\
\hline \multirow{10}{*}{ Cognitivo } & 1 & Pienso que aprender física es interesante. \\
\hline & 4 & Creo que aprender física es útil. \\
\hline & 7 & Opino que aprender física no es útil. \\
\hline & 10 & Considero que aprender física ayuda a entender otros temas. \\
\hline & 12 & Dudo que aprender física ayude a entender otros temas. \\
\hline & 14 & Creo que aprender física es necesario. \\
\hline & 16 & Pienso que aprender física es una pérdida de tiempo. \\
\hline & 18 & Considero que aprender física es difícil. \\
\hline & 20 & Me esfuerzo mucho para aprender física. \\
\hline & 22 & Resolver problemas es una pérdida de tiempo para aprender física. \\
\hline \multirow{8}{*}{ Afectivo } & 2 & Me desagrada aprender física. \\
\hline & 5 & Me interesa aprender física porque es interesante \\
\hline & 8 & Me aburre todo lo que esté relacionado con aprender física \\
\hline & 13 & Me siento feliz cuando no estudio física. \\
\hline & 15 & Detesto leer temas relacionados con física. \\
\hline & 17 & Me molesta tener que perder tiempo en aprender física. \\
\hline & 19 & Prefiero aprender cualquier otro curso menos aprender física. \\
\hline & 21 & Creo que aprender física no es aburrido \\
\hline \multirow{4}{*}{ Conductual } & 3 & Normalmente estudio para aprender física. \\
\hline & 6 & En clases me concentro cuando estoy aprendiendo física \\
\hline & 9 & Cuando aprendo física generalmente estoy atento en clases. \\
\hline & 11 & Me molesta aprender física porque no le encuentro sentido. \\
\hline
\end{tabular}

